Antagonists of the N-methyl-aspartate (NMDA) subtype of glutamate receptor induce neuronal degeneration confined to pyramidal neurons of rat corticolimbic brain areas. Previous findings have shown that NMDA receptor hypofunction and hyperactive muscarinic receptors produce a pattern of neuronal degeneration that resembles neurofibrillary pathology in AD. The objective of this study is to determine the role of caspase- 3 and calpain activation in the neuronal degeneration induced by MK-801 and pilocarpine administration. Using a monoclonal antibody that recognizes the holoprotein as well as fragments generated by caspase- 3 and calpain mediated cleavage of spectrin, we determined by Western-blotting a significant accumulation of caspase- 3 and calpain cleaved spectrin proteolytic fragments in the retrosplenial, entorhinal, pririfom cortex and amygdala of MK-801 (2.5, 5.0 and $10.0 \mathrm{mg} / \mathrm{Kg}$ (i.p.) plus pilocarpine $(10 \mathrm{mg} / \mathrm{kg}$ (s.c)) treated female SD rats relative to weight and age-matched saline treated controls at day 4 after drug treatment. Adminstration of MK-801 and pilocarpine increased the cleavage of spectrin by caspase- 3 and calpain proteases. These findings suggest that NMDA receptor hypofunction induces a feed forward loop of a sustained disinhibition of excitatory pathways as a result of inactivation of inhibitory neurons that results in a prolonged hyperstimulation of corticolimbic neurons that may involve cleavage of the cytoskeletal protein spectrin by calpain and caspase- 3 activation. Breakdown of spectrin may lead to a disruption of intraneuronal transport and consequent neuronal cell death.

\section{P2-024 PROTEIN MISFOLDING DONE RIGHT: THE BIOGENESIS OF BACTERIAL AMYLOID FIBERS}

Matthew Chapman, University of Michigan, Ann Arbor, MI, USA.

Contacte-mail: chapmanm@umich.edu

Background: We found that many Enterobacteriaceae spp., including E. coli, produce surface-localized amyloid fibers called curli. The dedicated amyloid machinery in E. coli provides a sophisticated and powerful model system for which to understand the fundamental principles of amyloid formation. Curli fibers are associated with biofilm formation, host cell adhesion and invasion, and immune system activation. Curli biogenesis is a directed and highly regulated process. The proteins required for curli biogenesis are encoded by the $\operatorname{csg} B A$ and $\operatorname{csg} D E F G$ operons. Objective: To utilize the power of $E$. coli genetics in order to understand amyloid formation. Methods: We have dissected the molecular activities of the curli proteins utilizing in vitro and in vivo techniques. The CsgE, CsgF, and $\mathrm{CsgG}$ proteins were found to be necessary for efficient, in vivo polymerization of major curlin subunit, CsgA, into amyloid fibers. In the absence of $\mathrm{CsgF}$, CsgA was secreted from the cell in a soluble, unpolymerized form. CsgA was unstable and degraded in the absence of the CsgE and $\mathrm{CsgG}$ proteins. Antibiotic sensitivity and protein secretion assays indicated that $\mathrm{CsgG}$ formed part of a curli-specific secretion apparatus in the outer membrane. High-resolution electron microscopy analysis demonstrated that $\mathrm{CsgG}$ exists as an oligomeric complex with an apparent central pore. Furthermore, we have investigated the polymerization kinetics of CsgA into an amyloid fiber in vitro. We have found that CsgA polymerizes with a nucleation dependent mechanism and that preformed CsgA fibers seed soluble CsgA polymerization. In vivo, CsgA is nucleated into an amyloid fiber by the $\mathrm{CsgB}$ protein. $\mathrm{CsgB}$ has amyloid-like characteristics and we propose a structural model of bacterial nucleation in which $\mathrm{CsgB}$ provides an amyloid template that guides the polymerization of CsgA. Conclusions: The dedicated amyloid machinery in E. coli provides a relevant and powerful system for which understand the fundamental principles of amyloid formation.

\section{P2-025 COPPER DEPLETION BY MENKES ATP7A OVER- EXPRESSION DRAMATICALLY ALTERS THE METABOLISM OF APP AND APLP2 IN CELL CULTURE}

Michael A. Cater ${ }^{1}$, Andrew Tsatsanis ${ }^{1}$, Irene Volitakis ${ }^{1}$, Qiao-Xin $\mathrm{Li}^{2}$, Genevieve Evin ${ }^{2}$, Julian F.B. Mercer ${ }^{3}$, Colin L. Masters ${ }^{1,2}$,
Robert A. Cherny ${ }^{1,2}$, Ashley I. Bush ${ }^{1,4},{ }^{I}$ Mental Health Research Institute of Victoria, Melbourne, Australia; ${ }^{2}$ The University of Melbourne, Melbourne, Australia; ${ }^{3}$ Deakin University, Melbourne, Australia; ${ }^{4}$ Genetics \& Aging Research Unit, Massachusetts General Hospital, Charlestown, MA, USA. Contacte-mail: mcater@mhri.edu.au

Background: In the cerebral amyloid plaques in Alzheimer's disease (AD), copper and zinc concentrations are enriched $(\sim 300 \%)$. Both these metals (but no other metals) directly coordinate to $\mathrm{A} \beta$ subunits (via histidine side chains) and co-purify with $\mathrm{A} \beta$ from post-mortem brains (Lovell et al 1998 J. Neuro. Sci.; Opazo et al 2002 J. Biol. Chem.). In addition, copper and zinc chelators have been shown to dissolve $\mathrm{A} \beta$ in post-mortem brains of patients (Cherny et al $1999 \mathrm{~J}$. Biol. Chem). Recent studies have also indicated that there may be a physiological role for APP or $\mathrm{A} \beta$ in exporting copper from neurons: APP knockout mice have elevated levels of copper in the brain (White et al 1999 Brain. Res), whereas APP transgenic mice (over-expressing APP) have decreased brain copper levels (Maynard et al 2002 J. Biol. Chem; Phinney et al 2003 PNAS). Objective: To modulate intracellular copper levels by manipulating the expression level of the cellular copper-efflux protein ATP7A and to investigate the effects upon APP and APLP2 transcript levels and cleavage. Methods: Vector-mediated ATP7A over-expression was achieved in human fibroblasts and neuroblastoma cell lines (SY5Y and M17). A fibroblast line derived from a Menkes disease patient (X-linked ATP7A mutant) was used to investigate the effect of intracellular copper accumulation on APP and APLP2 transcript levels and cleavage. Results and Conclusions: In cells over-expressing ATP7A (which causes depletion of copper), APP and APLP2 mRNA transcript could not be detected. These cells exhibited a marked decrease in intracellular APP levels, and APP secreted into the media appeared to shift completely from $\alpha$ - to $\beta$ - cleaved. Conversely, full length APLP2 levels were markedly increased and cleavage products decreased. ATP7A-mediated depletion of copper also changed the Western blot profile for BACE1 and presenilin 1. The Menkes patient fibroblasts (which accumulate copper) exhibited a small increase in intracellular APP and a striking increase in the amount of $\operatorname{sAPP} \alpha$ in comparison to normal fibroblasts. Taken together, these results indicate that intracellular copper levels are an import factor in modulating APP cleavage and expression.

\section{P2-026 THE CHOLESTEROL TRANSPORTER ABCG1 MODULATES THE PROTEOLYTIC PROCESSING OF AMYLOID PRECURSOR PROTEIN}

Gavin Tansley ${ }^{1}$, Braydon L. Burgess ${ }^{1}$, Matt Bryan ${ }^{2}$, Yuan $\mathrm{Su}^{2}$, Jeniffer Y. Chan ${ }^{1}$, Kathryn E. Naus ${ }^{1}$, Sean McIsaac ${ }^{1}$, Veronica Hirsch-Reinshagen ${ }^{1}$, Hsui-Chiung Yang ${ }^{1}$, Ronald B. DeMattos ${ }^{2}$, Cheryl L. Wellington ${ }^{1},{ }^{1}$ University of British Columbia, Vancouver, BC, Canada; ${ }^{2}$ Lilly Research Laboratories, Indianapolis, IN, USA. Contact e-mail: gtansley@cmmt.ubc.ca

Extracellular deposits of aggregated amyloid beta peptides (A $\beta$ ) derived from the proteolytic processing of amyloid precursor protein (APP) are a neuropathological hallmark of Alzheimer's Disease (AD). The cleavage of APP by secretases is modulated by the level and distribution of intracellular cholesterol. However, the influence of genes involved in lipid trafficking on the processing of APP remains poorly understood. The cholesterol transporter ABCG1 regulates the distribution and efflux of cholesterol and is highly expressed in the brain. Notably, the human ABCG1 gene maps to chromosome 21q22.3 and is inherited in excess in Down Syndrome (DS). Because individuals with DS typically manifest with AD neuropathology in their mid to late 30s, inheritance of excess ABCG1 may contribute to the accelerated onset of $\mathrm{AD}$ in DS. Here we show that expression of ABCG1 in HEK-APPswe cells results in increased cholesterol efflux and an accelerated production of $\mathrm{A} \beta 40$ and $\mathrm{A} \beta$ 42. Mutation of the conserved $\mathrm{ABC}$ signature sequence in ABCG1 blocks cholesterol efflux and reduces $A \beta$ secretion to baseline levels. Western blotting and biotinylation experiments reveal that ABCG1-expressing cells have increased total and cell-surface associated APP, as well as an increase in 